\title{
Asymptomatic cases of coronavirus disease 2019 (COVID-19):A review of literature on clinical characteristics and implications for epidemic control
}

\author{
Yanmeng Kang ${ }^{1}$, Ruiping Ma ${ }^{1}$, Yunxia Wang ${ }^{1}$, Degan $\mathrm{Lu}^{1}$, Chuanjun Huang ${ }^{1}$, Lingxia \\ Meng ${ }^{1}$, Mingxia $\mathrm{Gao}^{1}$, and Caiqing Zhang ${ }^{1}$ \\ ${ }^{1}$ Affiliation not available
}

October 8, 2020

\begin{abstract}
At present, the epidemic of Coronavirus Disease 2019 (COVID-19) is spreading worldwidely. Humans may be compelled to live with the viruses for a long time. The asymptomatic infections are contagious and the most difficult to control, easy to cause the accumulation of infection sources in the community. More and more asymptomatic cases have brought great challenges to the control of the epidemic. This review provides relatively comprehensive and updated information about asymptomatic cases of COVID-19. We find most of asymptomatic carriers are young people, and there are more asymptomatic infections in children compared with adult patients. Some asymptomatic carriers especially children have a longer communicable period than the confirmed cases. There is a risk of reoccurrence of positive result of nucleic acid test in asymptomatic cases. The chest CT findings of asymptomatic infections were mainly GGO, mostly peripheral distribution and restored quickly. Aerosol and fomite transmission of SARS-CoV-2 by asymptomatic carriers is plausible, so every person releases potentially infectious aerosols all the time. All countries in the world should raise public awareness of protection and strengthen social security measures to prevent second outbreak caused by the asymptomatic infections.
\end{abstract}

\section{Introduction}

Since the emergence of coronavirus disease 2019 (COVID-19) which is caused by severe acute respiratory syndrome coronavirus 2 (SARS-CoV-2) in December 2019, Wuhan, China, the epidemic spread around the world quickly. To date, the global COVID-19 pandemic has involved over 200 countries/regions, resulting in more than 7,000,000 confirmed cases and more than 400,000 deaths worldwide according to the real-time statistics of worldometer.

Many unknowns about COVID-19 remain, but we know clearly: it is both deadly and highly contagious. The estimated mean basic reproductive values $\left(\mathrm{R}_{0}\right)$ of COVID-19 is around 2 to 3 in the early phase of outbreak, which was higher than that of SARS and MERS[1,2]. Some scholars believe that the outbreak of any infectious disease follows such a rule: in the early stage severe cases are recognized first and then mild or asymptomatic cases are detected more frequently[3]. Asymptomatic carriers are hidden, difficult to be diagnosed and isolated in time, and easy to cause the accumulation of infection sources in the community[4]. So identification of asymptomatic

cases is very important for the control of COVID-19 epidemic.

Now we conducted a literature review, focusing on the age trend, nucleic acid test results, chest CT images, transmission modes, children asymptomatic cases, and the estimated proportion of asymptomatic infections of COVID-19. This review will be helpful to understand the unique features of the asymptomatic cases of COVID-19 and improve our epidemic prevention and control measures in the future. 
Asymptomatic cases were diagnosed based on positive SARS-CoV-2 viral nucleic acid test results but without any clinically detectable signs or self-perceived symptoms (e.g. fever,

sore throat, dry cough). According to the definition of CCDC (China Center for Disease Control), There are two types of asymptomatic persons: 1) those who have no self- perceived symptoms or clinically detectable signs throughout the 14-day quarantine; and 2) those who are "asymptomatic" during the incubation pe$\operatorname{riod}[4][5]$. In this article, we will mainly focus on the first type.

\section{Age trend}

A survey report on the epidemiological characteristics of COVID-19 cases from January 21 to February 20, 2020 in Zhejiang Province,China, indicated that in 108 asymptomatic cases, patients $; 30$ years old accounted for $28.70 \%$, higher than the ratio of the confirmed cases(13.86\%) [6]. Another investigation of imported cases from March 1 to April 7 from the same place showed that 43 cases were asymptomatic carriers in total 91 cases reported(47.25\%), and the proportion of patients; 30 years old in asymptomatic cases was $51.16 \%$, while it was $33.33 \%$ in the confirmed cases [7]. In Hunan province, China, the median ages of the confirmed cases and asymptomatic carriers of COVID-19 reported were 44 and 35 years respectively, and the proportion of young patients aged 3-18 years was proved to be relatively higher in asymptomatic cases $\left(\chi^{2}=65.52\right.$, $\left.\mathrm{P}_{j} 0.01\right)$ [8]. From January 21 to February 29, there were 14 cases of asymptomatic COVID-19 infection in Yangzhou, Jiangsu province, China, the majority of them were young adults, $85.7 \%$ of them were under 50 years old and the average age was 38.7 years[9].

Although some of the sample size was relatively small, the surveys were all from provinces located close to Hubei where the outbreak first started and the epidemic was severe, so we believed the results have some underestimated value. Moreover, We have reviewed literatures on family aggregation epidemic and list the asymptomatic carriers or close contacts without infection(no clinical symptoms and negative nucleic acid test), and found that women and children make up the majority of these cases(13/16, 81.25\%)(Table 1.) [10-21]. It is interesting that the six uninfected close contacts cases were all young females or children, none was adult male, though our sample size is very small(Table 1.). Several researches have showed that children are not susceptible to SARS-CoV-2 infection compared with the adults[22, 23], which still needs a large-scale epidemiological investigation to confirm it. We will talk about the children asymptomatic cases in this article later.

\section{Nucleic acid test results}

An analysis conducted by the CDC of Yangzhou, Jiangsu Province, China. followed up 14 asymptomatic cases and 23 confirmed cases of COVID-19 for six weeks. The results of virus nucleic acid test of respiratory tract specimen in the $2 \mathrm{nd}$, 3rd and 4th week after exposure showed that the positive rates of asymptomatic cases were $100.00 \%, 66.67 \%$ and $20.00 \%$, while in the confirmed group were $100.00 \%, 61.54 \%$ and $8.33 \%$ respectively. The positive rate fall to $0 \%$ in the 5 th week in the confirmed group, while $0 \%$ appear in the 7 th week in the asymptomatic cases. The researchers concluded that the positive rate was high in the 2 nd week and decreased gradually in both groups, but it lasted longer in asymptomatic cases than in confirmed cases and the difference was statistically significant $(\mathrm{P}<0.01)$. Meanwhile, the average values of $\mathrm{Ct}$ of the novel

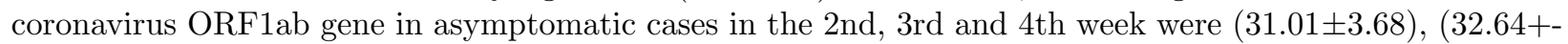
$3.74)$ and (29.05+-2.96), the $\mathrm{N}$ gene's were (31.37+-3.62), (32.90+-3.51) and (28.40+-4.19) respectively, and there showed no significant differences $(\mathrm{P}=0.23,0.12)[24]$. We think the small sample size makes the research results have some limitations.

Another question we focused on was the communicable period, which was defined as the duration of a positive nucleic acid test. Hu et al. reported the communicable period ranged from 1 to 21 days (median:9.5 days, IQR: 3.5-13.0 days)[25], while Li et al. concluded it ranged from 8 to 21 days (average:10.9 days)[9]. They both believe children asymptomatic carriers have a longer communicable period. So far, no detection methods could be able to accurately determine contagious period of COVID-19. The initial RT-PCR falsenegative rates were even up to $29 \%[26]$, which may be ascribed to the viral infection "window period", 
position of the sample collection and the experience of the operator and so on. Combination SARS-CoV-2 RNA test of different specimens with multiple detective methods such as a IgM-IgG combined antibody test or the next generation sequencing(NGS)may help to identify more asymptomatic carriers.

It has already been confirmed the viral load detected in the asymptomatic carriers was similar to that in the symptomatic patients[27], and many reports have confirmed that asymptomatic infections are contagious[14, 20, 28-32]. Hu et.al reported an asymptomatic carrier transmitted the virus to his family close contacts, and one of whom developed severe COVID-19 pneumonia monitored in ICU[25]. Some researchers also found that there is a risk of reoccurrence of positive result of nucleic acid test in asymptomatic cases after two negative results as well as in confirmed cases[24][33]. Understanding the implications of virus transmission in asymptomatic carriers is critical to developing containment strategies.

\section{Chest CT images}

Chest CT scan could offer reliable evidence for the detection of SARS-CoV-2 infection. Japanese scholar Inui et.al conduct a study on the chest CT of 112 cases from the cruise ship "Diamond Princess" with COVID-19[34]. They found 73\% (82/112) were asymptomatic infections, 54\% (44/82) of the asymptomatic cases had lung opacities on CT. They concluded that asymptomatic cases showed more GGO(ground-glass opacity)than consolidation and milder extension of lung parenchymal opacities. Another study is from the Central Hospital of Wuhan, China. Wang et al. make a retrospective analysis on the CT images from 159 asymptomatic cases with COVID-19 and found that the lesions in the lung were mostly peripheral distribution in 142 cases (89.3\%) and bilateral distribution in 91 cases (57.2\%), as well as more than two lung lobes were involved in 100 cases (62.9\%). Most of the lesions were GGO (95\%). In short-term follow-up, the majority of asymptomatic patients had a good prognosis $(73.7 \%)$ and none of them had extrapulmonary complications[35]. Hu et al. present the characteristics of chest CT images of 24 asymptomatic cases (but 5 cases developed symptoms later) and found that 8/19 cases showed ground-glass or patchy shadows, 4/19 cases showed stripe shadows which seems as an atypical image finding, and the rest 7/19 cases showed normal images[25].

These studies found that the chest CT images of asymptomatic cases were mainly GGO and most of them restored quickly, even though the sample size was small. However, the first two surveys did not indicate whether any of these asymptomatic cases developed symptoms later and what was the prognosis with different chest CT lesions. We expect more data on chest CT findings of the full-course asymptomatic carriers.

\section{Transmission modes}

How do asymptomatic carriers make the SARS-CoV-2 virus transmission and infect others?Because they do not cough or sneeze to any appreciable extent by definition, the direct or indirect contact modes and aerosol transmission are considered as the main possible modes of transmission[36]. Public health messages have paid attention to the possibility of direct and indirect transmission such as by contaminated hands, stressing the importance of washing hands often and thoroughly. Now we need to focuse on aerosol transmission. During the SARS epidemic in 2003, it has been verified that these infected hospitalized patients emitted viable aerosolized virus into the air[37]. A recent resarch by Doremalen et al. demonstrated that "aerosol and fomite transmission of SARS-CoV-2 is plausible, since the virus can remain viable and infectious in aerosols for hours and on surfaces up to days" [38]. It had been confirmed long before that ordinary breathing and speech both emit large quantities of aerosol particles[39, 40]. So the asymptomatic or pre-infected individuals still generate aerosols even if they do not sneeze or cough. Other studies have found that vocalization can emits up to an order of magnitude more aerosol particles than breathing, and the louder one speaks, the more aerosol particles are produced[41, 42].

It has been concluded by some researchers that COVID-19 can be transmitted by a face-to-face conversation with an asymptomatic infection individual without no touch, which ascribed to a large number of exhaled particles released during breathing and speech and the high transmissibility of SARS-CoV-2[36]. But how do the virus titers in these aerosols change with time after infection or discharge, and what is the best way 
to avoid infection from the point of environmental aerodynamics? Scientists need collaborate and take up the current challenge presented by COVID-19. At the same time, more publicity is needed to inform the public that everyone releases potentially infectious aerosols all the time, not just when sneezing or coughing, so it is essential to avoiding close contact and wearing a mask with protective properties.

\section{Children asymptomatic cases}

A report from the Wuhan Children's Hospital which located in the outbreak and worst-hit areas

described the spectrum of SARS-CoV-2 infection in children, it analyzed 171 cases and concluded that most infected children appear to have a milder clinical course in contrast with infected adults, and asymptomatic cases $(27 / 171,15.8 \%)$ were more common[43]. A retrospective study of 2143

pediatric patients infected with SARS-CoV-2 in China come to a similar conclusion, the majority of the patients were asymptomatic(94, $4.4 \%$ ), mild (1091, 50.9\%), or moderate cases( $831,38.8 \%$ ), totally accounted for $94.1 \%$ of all cases. It suggests that there are fewer severe cases but more asymptomatic infections in children compared with adult patients[44].

Some scholars believe that this is due to less immune dysfunction after the SARS-CoV-2 infection in children[45-48]. It is very meaningful that vaccination with the tuberculosis vaccine bacillus Calmette Guerin (BCG) could dramatically reducing infant deaths from sepsis and respiratory tract infection (e.g. influenza). BCG has also been proved to protect mice from influenza virus infection via nonspecific protection in animal experiment[49]. This is a type of immune memory phenomenon that is unique to innate immunity after body is infected or vaccinated and it can induce $\mathrm{T}$ cell-independent protection against secondary infection, we call it "training immunity" [50]. Perhaps the helpful training immunity in asymptomatic or mild infection children could contribute to the future evolution of immunotherapy and vaccines for the SARS-CoV-2. Another speculation of why children were less sensitive to SARS-CoV-2 was that the function (e.g. binding ability) of Angiotensin Converting Enzyme-2 (ACE-2) identified as the cell entry receptor of SARS-CoV-2 in children may be lower than that in adults[44].

\section{The proportion of asymptomatic infections}

The epidemic of SARS-CoV-2 infection is spreading worldwidely, compeling us to live with the virus for perhaps a long time. From the prevention point of view, the asymptomatic carriers are the most difficult to control, because they spread the virus unknowingly on some occasions. Estimation of the prevalence of asymptomatic virus shedding such as asymptomatic ratio by using target populations could improve understanding of COVID-19 transmission and provide vital insight into the epidemic spread[51]. The figure can put severe cases and mortality rates into an accurate context so that the fatality rate and the proportion of patients requiring hospitalization would be estimated accurately, which is critical to balancing the socioeconomic burden of infection control interventions against their potential benefit for humans[52]. As of June 06, 2020, 7,938 asymptomatic infections had been confirmed on the mainland of China, including 1,358 re-categorized as confirmed cases, while total confirmed cases reached 83036[53]. At present, the proportion of asymptomatic infection in mainland China was $7.23 \%(6580 / 90974)$, seems lower than our estimation, which may be attributed to the following factors: incomplete screening, defective detection methods, and some patients have conscious symptoms due to psychological pressure and so on.

How many asymptomatic cases globally, it's hard to answer this question now. Japanese scholar estimated that the asymptomatic proportion of COVID-19 on board the Diamond Princess cruise ship was at $17.9 \%$ (95\%CI: $15.5-20.2 \%$ ) by conducting statistical modeling analyses[54]. Another assessment of the asymptomatic ratio among Japanese nationals who evacuated from Wuhan, China, got a higher outcome of $41.6 \%$ (95\% CI: $16.7 \%-66.7 \%)$. But the sample data of both studies that adopted in the analysis did not constitute a random sample of a general population, and there was a bias from the presence of underlying diseases or comorbidities of the samples, which brought some limitations. Taking results from several studies into account, Gerardo Chowell, a mathematical epidemiologist at Georgia State University in Atlanta, thinks 
that "asymptomatic or mild cases combined represent about $40-50 \%$ of all infections" [55]. To assess the extent of hidden infection, a group of researchers had developed a model using clinical data from 26,000 laboratory-confirmed cases reported to the health commission of Wuhan, the epicentre of the outbreak, China. "By our most conservative estimate, at least $59 \%$ of the infected individuals were out and about, without being tested and potentially infecting others," says Wu Tangchun who in charge of the project[55]. We look forward to the final detailed results yet not published.

\section{Conclusion}

Nowadays, Coronavirus Disease 2019 (COVID-19) is rampaging though the world. Finding the source of infection and cutting off transmission are important measures to control COVID-19 before the effective vaccine is developed. However, as the epidemic progressed overtime, more and more asymptomatic cases brought huge challenges to the control of COVID-19. This review provides relatively comprehensive and updated information about asymptomatic cases of COVID-19. Further research into the prevalence of asymptomatic infection of COVID-19 is necessary to guide epidemic control efforts. All countries in the world should raise public awareness of protection and strengthen social security measures to prevent second outbreak caused by asymptomatic infection.

\section{Funding source}

This research did not receive any specific grant from funding agencies in the public, commercial, or not-forprofit sectors.

\section{Ethical approval}

Not required any ethical approval for this study.

\section{Conflicts of interest}

The authors declare that they have no conflict of interest.

\section{References}

1. Wu J T, Leung K, and Leung G M, Nowcasting and forecasting the potential domestic and international spread of the 2019-nCoV outbreak originating in Wuhan, China: a modelling study. The Lancet, 2020.395 (10225): p. 689-697.

2. Liu Y, Gayle A A, Wildersmith A, and Rocklov J, The reproductive number of COVID-19 is higher compared to SARS coronavirus.Journal of Travel Medicine, 2020. 27 (2).

3. Al-Tawfiq J A, Asymptomatic coronavirus infection: MERS-CoV and SARS-CoV-2 (COVID-19). Travel Med Infect Dis, 2020.27 (101608): p. 1-2.

4. Novel C P E R E, The epidemiological characteristics of an outbreak of 2019 novel coronavirus diseases (COVID-19) in China.Zhonghua liu xing bing xue za zhi= Zhonghua liuxingbingxue zazhi, 2020.41 (2): p. 145.

5. Protocol for the Management of Asymptomatic Persons Infected with COVID-19 Virus.Joint Taskforce on COVID-19 Prevention and Control, China State Council

6. Junfen L, Mengna W, Haocheng W, Tao Z, Chen W, and Fudong L,Epidemiological characteristics of coronavirus disease 2019 in Zhejiang Province. Prev Med, 2020. 32 (03): p. 217-221+225.

7. Biyao L, Xiaohua Q, Min J, and Zhen W, Epidemiological characteristics of imported COVID-19 cases from aboard to Zhejiang Province Preventive Medicine, 2020: p. 1-9.

8. Hu S, Xu Q, Luo K, Yang H, Sun Q, Liu Z, et al.,Epidemiological characteristics of patients with coronavirus disease 2019 in Hunan province. Pract Prev Med, 2020. 27 (04): p. 385-388. 
9. Jincheng L, Qin X, Yan W, Jie X, Yao H, Wenjun L, et al.,Analysis in characteristics of asymptomatic infection patients with coronavirus disease 2019 in Yangzhou City of Jiangsu Province.Journal of Clinical Medicine in Practice, 2020. 24 (05): p. 10-13.

10. Pan X, Chen D, Xia Y, Wu X, Li T, Ou X, et al., Asymptomatic cases in a family cluster with SARSCoV-2 infection. The Lancet Infectious Diseases, 2020. 20 (4): p. 410-411.

11. Bai Y, Yao L, Wei T, Tian F, Jin D, Chen L, et al., Presumed asymptomatic carrier transmission of COVID-19. Jama, 2020.323 (14): p. 1406-1407.

12. Li P, Fu J, Li K, Chen Y, Wang H, Liu L, et al., Transmission of COVID-19 in the terminal stage of incubation period: a familial cluster. International Journal of Infectious Diseases, 2020.

13. Tong Z, Tang A, Li K, Li P, Wang H, Yi J, et al., Potential presymptomatic transmission of SARS-CoV-2, Zhejiang province, China, 2020. Emerging infectious diseases, 2020. 26 (5): p. 1052.

14. Liu X, Liu F, Fan Q, Zheng W, Zhang D H, Zhang Q L, et al.,Report of COVID-19 family clustering epidemic caused by asymptomatic infection. Chin J Public Health, 2020. 36 (03): p. 282-284.

15. Qian G, Yang N, Ma A H Y, Wang L, Li G, Chen X, et al.,COVID-19 Transmission Within a Family Cluster by Presymptomatic Carriers in China. Clinical Infectious Diseases, 2020.

16. Ma W, Su B, Wu J, Chen F, Bu T, Cheng X, et al., Report of COVID-19 family clustering epidemic caused by asymptomatic infection.Chin J Public Health, 2020. 36 (03): p. 277-281.

17. Tian Y, Wu Z, Han X, Wang Y, and Liu H, Epidemiological investigation on the first family aggregation epidemic of novel coronavirus pneumonia in Yangzhou Shanghai Journal of Preventive Medicine, 2020: p. 1-7.DOI: https://doi.org/10.19428/j.cnki.sjpm.2019.20117.

18. Liu G and Ma ZL, Family clustering of novel coronavirus disease 2019 in Liaoning province: a brief report. Chin J Public Health,, 2020.36 (03): p. 293-294.

19. Li C, Wu B, Luo F, and Zhang N, Clinical Study and CT Findings of a Familial Cluster of Pneumonia with Coronavirus Disease 2019 (COVID-19) J Sichuan Univ ( Med Sci Edi ), 2020. 51 (02): p. 155-158.

20. Ye F, Xu S, Rong Z, Xu R, Liu X, Deng P, et al., Delivery of infection from asymptomatic carriers of COVID-19 in a familial cluster.International Journal of Infectious Diseases, 2020.

21. Yang YL, Li LH , LI CF, YC X, and J P,A survey on clustering of novel coronavirus disease in families in Fengjie county of Chongqing municipality. Chin J Public Health, 2020.36 (03): p. 285-288.

22. Guan W, Ni Z, Hu Y, Liang W, Ou C, He J, et al., Clinical characteristics of coronavirus disease 2019 in China. New England journal of medicine, 2020. 382 (18): p. 1708-1720.

23. Xu Y, Li X, Zhu B, Liang H, Fang C, Gong Y, et al., Characteristics of pediatric SARS-CoV-2 infection and potential evidence for persistent fecal viral shedding. Nature medicine, 2020.26 (4): p. 502-505.

24. Dong Y, Fan Q, Wang Y, Li Y, Xia J, Yao J, et al., Analysis in nucleic acid test results of patients with coronavirus disease 2019 in Yangzhou City of Jiangsu Province. Journal of Clinical Medicine in Practice, 2020. 24 (05): p. 6-9.

25. Hu Z, Song C, Xu C, Jin G, Chen Y, Xu X, et al., Clinical characteristics of 24 asymptomatic infections with COVID-19 screened among close contacts in Nanjing, China. Science China Life Sciences, 2020: p. $1-6$.

26. Tan C, Xiao Y, Meng X, Huang X, Li C, and Wu A, Asymptomatic SARS-CoV-2 infections: what we need to know? Infection Control \& Hospital Epidemiology, 2020: p. 1-7.

27. Zou L, Ruan F, Huang M, Liang L, Huang H, Hong Z, et al.,SARS-CoV-2 viral load in upper respiratory specimens of infected patients. New England Journal of Medicine, 2020. 382 (12): p. 1177-1179. 
28. Rothe C, Schunk M, Sothmann P, Bretzel G, Froeschl G, Wallrauch C, et al., Transmission of 2019$n C o V$ infection from an asymptomatic contact in Germany. New England Journal of Medicine, 2020.382 (10): p. 970-971.

29. Lei M Y, Huang Y, Gou Y, Ping K K, Liu S B, Yao G H, et al., Characteristics of cluster epidemic of 2019 novel coronavirus disease in Guizhou province. Chin J Public Health, 2020: p. 1-5.

30. Liu Y C, Liao C H, Chang C F, Chou C C, and Lin Y R, A locally transmitted case of SARS-CoV-2 infection in Taiwan. New England Journal of Medicine, 2020. 382 (11): p. 1070-1072.

31. Lai C-C, Shih T-P, Ko W-C, Tang H-J, and Hsueh P-R, Severe acute respiratory syndrome coronavirus 2 (SARS-CoV-2) and corona virus disease-2019 (COVID-19): the epidemic and the challenges. International journal of antimicrobial agents, 2020: p. 105924.

32. Yu P, Zhu J, Zhang Z, and Han Y, A familial cluster of infection associated with the 2019 novel coronavirus indicating possible person-to-person transmission during the incubation period. The Journal of infectious diseases, 2020. 221 (11): p. 1757-1761.

33. Xuzhou municipal health commission. Notification of the epidemic (no.

http://www.xzcdc.com/index.php/news/15484.html.

34. Inui S, Fujikawa A, Jitsu M, Kunishima N, Watanabe S, Suzuki Y, et al., Chest CT findings in cases from the cruise ship "Diamond Princess" with coronavirus disease 2019 (COVID-19). Radiology: Cardiothoracic Imaging, 2020. 2 (2): p. e200110.

35. Wang Y, Chen J, and Wang X, CT image features of asymptomatic patients with novel coronavirus pneumonia. Medical Journal of Wuhan University, 2020. 41 (03): p. 353-356.

36. Asadi S, Bouvier N, Wexler A S, and Ristenpart W D, The coronavirus pandemic and aerosols: Does COVID-19 transmit via expiratory particles? 2020, Taylor \& Francis.

37. Booth T F, Kournikakis B, Bastien N, Ho J, Kobasa D, Stadnyk L, et al., Detection of airborne severe acute respiratory syndrome (SARS) coronavirus and environmental contamination in SARS outbreak units. The Journal of infectious diseases, 2005. 191 (9): p. 1472-1477.

38. van Doremalen N, Bushmaker T, Morris D H, Holbrook M G, Gamble A, Williamson B N, et al., Aerosol and surface stability of SARS-CoV-2 as compared with SARS-CoV-1. New England Journal of Medicine, 2020. 382 (16): p. 1564-1567.

39. JP D, The size and the duration of air-carriage of respiratory droplets and droplet-nuclei. Epidemiology \& Infection, 1946.44 (6): p. 471-479.

40. PAPINENI R S and ROSENTHAL F S, The size distribution of droplets in the exhaled breath of healthy human subjects. Journal of Aerosol Medicine, 1997. 10 (2): p. 105-116.

41. Morawska L, Johnson G, Ristovski Z, Hargreaves M, Mengersen K, Corbett S, et al., Size distribution and sites of origin of droplets expelled from the human respiratory tract during expiratory activities. Journal of Aerosol Science, 2009. 40 (3): p. 256-269.

42. Asadi S, Wexler A S, Cappa C D, Barreda S, Bouvier N M, and Ristenpart W D, Aerosol emission and superemission during human speech increase with voice loudness. Scientific reports, 2019.9 (1): p. 1-10.

43. Lu X, Zhang L, Du H, Zhang J, Li Y Y, Qu J, et al., SARS-CoV-2 infection in children. New England Journal of Medicine, 2020.382 (17): p. 1663-1665.

44. Dong Y, Mo X, Hu Y, Qi X, Jiang F, Jiang Z, et al.,Epidemiological characteristics of 2143 pediatric patients with 2019 coronavirus disease in China. Pediatrics, 2020.

45. Wei M, Yuan J, Liu Y, Fu T, Yu X, and Zhang Z-J, Novel coronavirus infection in hospitalized infants under 1 year of age in China. Jama, 2020. 323 (13): p. 1313-1314. 
46. Chen H, Guo J, Wang C, Luo F, Yu X, Zhang W, et al., Clinical characteristics and intrauterine vertical transmission potential of COVID-19 infection in nine pregnant women: a retrospective review of medical records. The Lancet, 2020. 395 (10226): p. 809-815.

47. Zeng L, Tao X, Yuan W, Wang J, Liu X, and Liu Z, First case of neonate infected with novel coronavirus pneumonia in China. Zhonghua er ke za zhi= Chinese journal of pediatrics, 2020. 58 : p. E009.

48. Cao Q, Chen Y, Chen C, and Chiu C, SARS-CoV-2 infection in children: Transmission dynamics and clinical characteristics. Journal of the Formosan Medical Association, 2020. 119 (3): p. 670.

49. JW V t W, R P, and $\mathrm{R} \mathrm{V} \mathrm{F,} \mathrm{The} \mathrm{role} \mathrm{of} B C G / P P D$-activated macrophages in resistance against systemic candidiasis in mice.Scandinavian journal of immunology, 1992. 36 (5): p. 713-720.

50. Netea M G, Schlitzer A, Placek K, Joosten L A, and Schultze J L, Innate and adaptive immune memory: an evolutionary continuum in the host's response to pathogens. Cell host \& microbe, 2019.25 (1): p. 13-26.

51. Nishiura H, Kobayashi T, Miyama T, Suzuki A, Jung S, Hayashi K, et al., Estimation of the asymptomatic ratio of novel coronavirus infections (COVID-19). medRxiv, 2020.

52. Battegay M, Kuehl R, Tschudin-Sutter S, Hirsch H H, Widmer A F, and Neher R A, 2019-novel Coronavirus (2019-nCoV): estimating the case fatality rate-a word of caution. Swiss medical weekly, 2020.150 (0506).

53. Information from the official website of the National Health Commissionhttp://www.nhc.gov.cn/xcs/xxgzbd/gzbd_index.shtml

54. Mizumoto K, Kagaya K, Zarebski A, and Chowell G, Estimating the asymptomatic proportion of coronavirus disease 2019 (COVID-19) cases on board the Diamond Princess cruise ship, Yokohama, Japan, 2020.Eurosurveillance, 2020. 25 (10): p. 2000180.

55. Qiu J, Covert coronavirus infections could be seeding new outbreaks. Nature, 2020.

\section{Hosted file}

Table 1.pdf available at https://authorea.com/users/365197/articles/485379-asymptomaticcases-of-coronavirus-disease-2019-covid-19-a-review-of-literature-on-clinical-

characteristics-and-implications-for-epidemic-control 